\title{
Black hole entropy as T-duality invariant
}

\author{
Kenji Suzuki \\ Department of physics, Tokyo Institute of Technology \\ Oh-okayama, Meguro, Tokyo 152, Japan \\ ks@th.phys.titech.ac.jp
}

\begin{abstract}
We study the Euler numbers and the entropies of the non-extremal intersecting D-branes in ten-dimensions. We use the surface gravity to constrain the compactification radii. We correctly obtain the integer valued Euler numbers for these radii. Moreover, the entropies are found to be invariant under the T-duality transformation. In the extremal limit, we obtain the finite entropies only for two intersecting D-branes. We observe that these entropies are proportional to the product of the charges of each D-brane. We further study the entropies of the boosted metrics. We find that their entropies can be interpreted in term of the microscopic states of D-branes.
\end{abstract}

04.70.Dy, 04.20.Gz, 04.50.+h 


\section{INTRODUCTION}

The Bekenstein-Hawking entropies of the BPS saturated black holes have been studied in four-, five- and ten-dimensions from the nonperturbative aspects of string theory [1 8 . The black holes in ten-dimensions are constructed by the intersecting D-branes. The black holes are dual to each other under the T-duality transformation. The four- and five-dimensional black holes are obtained by dimensional reduction from the ten-dimensional black holes. The method to obtain the non-extremal black holes from the extremal black holes are studied. Their entropies are interpreted in terms of the microscopic states. The effect of the graybody factor to the Hawking-radiate blackbody radiation is also derived from the D-branes.

On the other hand, the Euler numbers of the black holes are investigated in fourdimensions [9 11]. The Euler number $\chi$ of an $n$-dimensional manifold is the sum of the Betti numbers $B_{p}$ :

$$
\chi=\sum_{p=0}^{n}(-1)^{p} B_{p} .
$$

The Betti numbers are integers. Therefore the Euler numbers are also integers. We can also calculate the Euler numbers by using the $n$-dimensional Gauss-Bonnet action, which integrates the curvatures $R_{i j}$ of the black holes. The $n$-dimensional Gauss Bonnet action is

$$
\chi=\frac{1}{(4 \pi)^{n} n !} \int d x^{n} \epsilon^{i_{1} i_{2} \cdots i_{n}} R_{i_{1} i_{2}} R_{i_{3} i_{4}} \cdots R_{i_{n-1} i_{n}} .
$$

The black holes in four-dimensions have the period in Euclidean time coordinate. The period is defined by the inverse of the surface gravity. By using this relation, we can obtain the integer valued Euler numbers.

In the same way as in four-dimensions, we can calculate the Euler numbers of the black holes in ten-dimensions using the ten-dimensional Gauss-Bonnet action. The Euler numbers of the black holes in ten-dimensions have not been studied so far. We consider the Euler numbers of the black holes which are realized by the intersecting D-branes in ten-dimensions. The black holes of the intersecting D-branes in ten-dimensions also have the periods with respect to the compactification radii. The radii are usually taken to be arbitrary. For the generic radii, the Euler numbers calculated by the ten-dimensional Gauss-Bonnet actions are non-integers. However the Euler numbers must be integers because of their definition. To avoid these difficulties, we propose a way to constrain the compactification radii.

We reflect on the necessity to fix the values of the compactfication radii in the physical reasoning. We treat the black holes in ten-dimensions. These have six compactified directions. The entropies of the black holes in ten-dimensions are expressed by the compactification radii and the charges. We rewrite the entropies using the quantized charges and the Newton's constant, then the entropies are irrespective of the compactification radii. Therefore it seems not to be necessary to fix the value of the compactification radii. However, the four-dimentional Newton's constant $G_{(4)}$ depends on the value of the compactified radii,

$$
G_{(4)}=G_{(10)} / L_{1} L_{2} L_{3} L_{4} L_{5} L_{6}
$$


where $L_{i}$ are the compactification radii. $G_{(10)}$ is the ten-dimensional Newton's constant,

$$
G_{(10)}=8 \pi^{6} g^{2}
$$

with $\alpha^{\prime}=1$. We find that the compactified radii depend on the radius of the horizon $(\mu)$ and the charges, using the method of the surface gravities of the compactified directions, discussed in the section 3. Then the four-dimensional Newton's constant is also rewritten by $\mu$ and the charges. We are interested in the behavior of the four-dimensional Newton's constant in the BPS limit, $\mu \rightarrow 0$. As a result, we obtain that the Newton's constant vanishes in the BPS limit.

The another physical reasoning that we need to fix the compactification radii is to avoid the singular effects of the horizon in the compactified directions. We recall the way to define the period in the time direction. If no singular effects of the horizon exist in $t-r$ directions, then the topology of these directions are $\mathbf{R}^{\mathbf{2}}$, and the Euler number of these directions is 1. Using the Gauss-Bonnet theorem, we find that the Euclidean time coordinate have the period which is the inverse of the surface gravity. Therefore we need to take this period in the time direction to avoid the singular effects of the horizon. Similarly, we need to fix the compactification radii to avoid the singular effects of the horizon in the compactified directions. We find that the compactification radii are the inverses of the surface gravity in the compactified directions using the Gauss-Bonnet theorem. We discuss this point in the section 3 .

The purpose of this paper is to constrain the compactification radii in order to obtain the integer valued Euler numbers for the non-extremal black holes of the intersecting D-branes in ten-dimensions. We further study the microscopic interpretations of their entropies.

We consider the black holes of the intersecting D-branes which have some compactification radii in ten-dimensions [2,66, 7]. We assume that the metrics only depend on $r=\sqrt{x^{2}+y^{2}+z^{2}}$. In the four-dimensions, the black holes have the period in Euclidean time coordinate. The period is defined by the inverse of the surface gravity. We extend this construction to constrain the compactified radii in ten-dimensions. We use the inverse of the surface gravities in the compactified directions to constrain these radii. As a result, we are able to obtain the integer Euler numbers. We recall that the temperatures are defined by the surface gravities in the time coordinates. The relation between the radii and the surface gravities generalizes the temperatures of the black holes.

Using these results, we further obtain the entropies of the non-extremal intersecting Dbranes. These entropies are invariant under T-duality transformation. In the BPS limit, we obtain the finite and non-vanishing entropies only for two intersecting D-branes. We observe that they can be interpreted as the product of charges of each D-brane. We then study the entropies of the boosted metrics. We obtain the famous relation between the entropies and the quantized D-brane charges and the internal momenta of the intersecting D-branes. These relations are the same as that of the microscopic D-brane picture [3] 5].

The organization of this paper is as follows. In section 2, we review the way to construct the intersecting D-branes of the type IIA and IIB superstrings. We explain how to obtain the non-extremal black holes from the extremal black holes. In addition we define the boost transformation of the metrics. In section 3, we consider the surface gravities in the compactified directions to constrain the compactification radii. As a result, we obtain the Euler numbers which are integers. In section 4, we calculate the entropies of the non- 
extremal intersecting D-branes and study them in the BPS limit. We further discuss the interpretation of these entropies in terms of the microscopic states.

\section{NON-EXTREMAL INTERSECTING D-BRANES IN TEN-DIMENSIONS}

In this section we review the way to construct the intersecting D-branes [6]. We then explain how to obtain non-extremal black holes from the extremal black holes and to obtain the boosted metrics. We treat the intersecting D-branes in ten-dimensions with the three-dimensional transverse directions with respect to the D-branes. This means that the harmonic functions of these metrics only depend on $r=\sqrt{x^{2}+y^{2}+z^{2}}$.

We introduce the metrics and the field strengths in association with the D-branes. The metrics of the $\mathrm{D} n$-branes wrapped on the $i_{1}, i_{2}, \cdots i_{n^{-}}$directions are

$$
d s_{10}^{2}=H^{1 / 2}\left[H^{-1}\left(-d t^{2}+d x_{i_{1}}^{2}+\cdots+d x_{i_{n}}^{2}\right)+d r^{2}+r^{2} d \Omega_{10-n-2}^{2}\right] .
$$

$H$ is the harmonic function. The field strengths are

$$
\mathcal{F}= \begin{cases}d t \wedge d H^{-1} \wedge d x_{i_{1}} \wedge \cdots \wedge d x_{i_{n}} & (n \leq 3 ; \text { electric }) \\ *\left(d t \wedge d H \wedge d x_{i_{1}} \wedge \cdots \wedge d x_{i_{n}}\right) & (n \geq 3 ; \text { magnetic }),\end{cases}
$$

where $*$ is the Hodge dual in ten-dimensions. In $n=3$, the field strength is self-dual. Then they have the field strengths both of electric and magnetic nature. For the intersecting D-branes, $\mathcal{F}$ are the sum of the field strengths of each D-brane. We obtain the metrics of the type IIA string theory from the intersecting M-branes by dimensional reductions. The intersecting M-branes with the three-dimensional transverse directions with respect to the M-branes are $(2,2,5,5)_{M},(5,5,5)_{M},(2,5,5)_{M},(2,2,5)_{M}$, and $(5,5)_{M}$, where $(5,5)_{M}$ denote two intersecting M5-branes, and so on [2, 6, 7].

We subsequently review the metrics of three intersecting D-branes. In ten-dimensions, we can obtain the $(4,4,4)$ metric from the intersecting M-branes $(5,5,5)_{M}$ upon dimensional reduction along a common direction of three intersecting M5-branes :

$$
\begin{aligned}
d s_{10}^{2}= & \left(H_{1} H_{2} H_{3}\right)^{1 / 2}\left[-H_{1}^{-1} H_{2}^{-1} H_{3}^{-1} d t^{2}\right. \\
& +H_{1}^{-1} H_{2}^{-1}\left(d x_{1}^{2}+d x_{2}^{2}\right)+H_{1}^{-1} H_{3}^{-1}\left(d x_{3}^{2}+d x_{4}^{2}\right) \\
& \left.+H_{2}^{-1} H_{3}^{-1}\left(d x_{5}^{2}+d x_{6}^{2}\right)+d r^{2}+r^{2} d \Omega_{2}^{2}\right] .
\end{aligned}
$$

The field strength $\mathcal{F}_{4(4,4,4)}$ and the dilaton $\phi$ are

$$
\begin{aligned}
\mathcal{F}_{4(4,4,4)}= & \left(\partial_{r} H_{1} d \theta \wedge d \phi \wedge d x_{6} \wedge d x_{7}\right. \\
& \left.+\partial_{r} H_{2} d \theta \wedge d \phi \wedge d x_{4} \wedge d x_{5}+\partial_{r} H_{3} d \theta \wedge d \phi \wedge d x_{1} \wedge d x_{2}\right) \\
e^{-2 \phi}= & \left(H_{1} H_{2} H_{3}\right)^{1 / 2}
\end{aligned}
$$

where $H_{i}=1+\frac{Q_{i}}{r}$. We denote this metric as the following [8]:

$$
(4,4,4)=\left\{\begin{array}{l|llllllll}
\times & \times & \times & \times & \times & - & - & - & - \\
\times & \times & \times & - & - & \times & \times & - & - \\
\times & - & \times & \times & \times & \times & - & - & -
\end{array} .\right.
$$


Similarly we can obtain the $(2,4,4)$ metric, from $(2,5,5)_{M}$ upon dimensional reduction along a direction of two intersecting M5-branes,

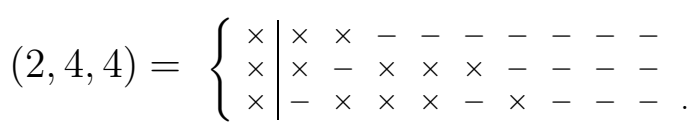

The field strength $\mathcal{F}_{4(2,4,4)}$ and the dilaton $\phi$ are

$$
\begin{aligned}
\mathcal{F}_{4(2,4,4)}= & \left(d t \wedge \partial_{r} H_{1}^{-1} d r \wedge d x_{1} \wedge d x_{2}\right. \\
& \left.+\partial_{r} H_{2} d \theta \wedge d \phi \wedge d x_{2} \wedge d x_{6}+\partial_{r} H_{3} d \theta \wedge d \phi \wedge d x_{1} \wedge d x_{5}\right) \\
e^{-2 \phi}= & \left(H_{1}^{-1} H_{2} H_{3}\right)^{1 / 2}
\end{aligned}
$$

Along a direction of the M5-brane, we can obtain the $(2,2,4)$ metric,

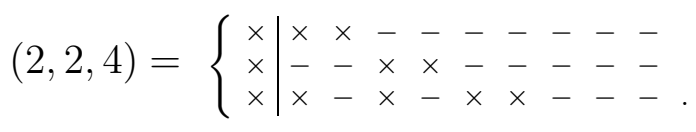

The field strength $\mathcal{F}_{4(2,2,4)}$ and the dilaton $\phi$ are

$$
\begin{aligned}
\mathcal{F}_{4(2,2,4)}= & \left(d t \wedge \partial_{r} H_{1}^{-1} d r \wedge d x_{1} \wedge d x_{2}\right. \\
& \left.+d t \wedge \partial_{r} H_{2}^{-1} d r \wedge d x_{3} \wedge d x_{4}+\partial_{r} H_{3} d \theta \wedge d \phi \wedge d x_{2} \wedge d x_{4}\right), \\
e^{-2 \phi}= & \left(H_{1} H_{2} H_{3}^{-1}\right)^{-1 / 2}
\end{aligned}
$$

In these constructions, we have regarded the D2-branes as electric and the D4-branes as magnetic objects.

Moreover, we can obtain the remaining metrics by T-duality transformation from these metrics in the $i$-th direction [7] :

$$
g_{i i}^{\prime}=1 / g_{i i}, \quad \exp \left(-2 \phi^{\prime}\right)=g_{i i} \exp (-2 \phi) .
$$

In our notation, the T-duality transformation is realized by interchanging $\times$ and - . For example, in the three intersecting D-branes case,

$$
\left\{\begin{array} { l } 
{ x } \\
{ x } \\
{ - }
\end{array} \leftrightarrow \left\{\begin{array}{l}
- \\
- \\
x
\end{array}\right.\right.
$$

If we apply the T-duality transformation on the metric $(4,4,4)$ (in (5)) in the first direction, this metric is changed to $(3,3,5)$. Similarly, we can obtain the following metrics :

$$
\begin{aligned}
& (4,4,4) \leftrightarrow(3,3,5) \leftrightarrow(2,4,4) \text { or }(2,2,6) \\
& \leftrightarrow(3,3,3) \text { or }(1,3,5) \leftrightarrow(2,2,4) \text { or }(0,4,4) \\
& \leftrightarrow(1,3,3) \leftrightarrow(2,2,2) .
\end{aligned}
$$

The metrics of the intersecting even-branes are the metrics of type IIA. The metrics of the intersecting odd-branes are the metrics of type IIB.

For four intersecting D-branes, we obtain the following metric: 


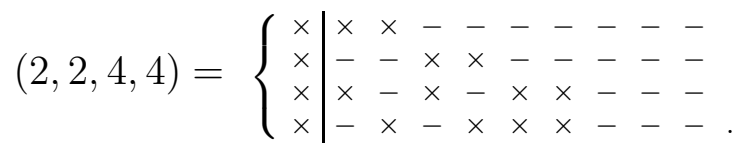

from $(2,2,5,5)_{M}$. Applying the T-duality transformation, we can obtain the following metrics :

$$
(3,3,3,3) \leftrightarrow(2,2,4,4) \leftrightarrow(1,3,3,5) \leftrightarrow(0,4,4,4)
$$

For two intersecting D-branes, we obtain the $(4,4)$ metrics:

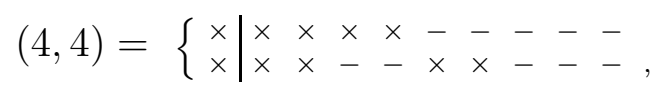

from $(5,5)_{M}$. Applying the T-duality transformation, we can obtain following metrics :

$$
\begin{aligned}
&(4,4) \leftrightarrow(3,5) \leftrightarrow(2,6), \\
&(3,3) \leftrightarrow(2,4) \leftrightarrow(1,5), \\
&(2,2) \leftrightarrow(1,3) \leftrightarrow(0,4),
\end{aligned}
$$

in a non-intersecting direction, and

$$
(4,4) \leftrightarrow(3,3) \leftrightarrow(2,2)
$$

in an intersecting direction. We have assumed that these metrics have the three-dimensional transverse directions. Therefore all harmonic functions in these metrics only depend on $r=\sqrt{x^{2}+y^{2}+z^{2}}$. Note that our notation is different from [8].

These metrics can also be obtained from the metrics of three intersecting D-branes. For example, we find the following relation:

$$
(3,3,5) \rightarrow(3,3)
$$

by assuming the third D5-brane's charge to vanish. For single D-brane, the T-duality transformation implies the following relation

$$
(6) \leftrightarrow(5) \leftrightarrow \cdots \leftrightarrow(0)
$$

In order to obtain non-extremal metrics, we modify the above metrics as follows [2] :

(1) We make the following replacements in the transverse space-time part of the metric:

$$
d t^{2} \rightarrow f(r) d t^{2}, \quad d r^{2} \rightarrow f^{-1}(r) d r^{2}, \quad f(r)=1-\frac{\mu}{r}
$$

We also use the harmonic functions,

$$
H_{i}=1+\frac{\mathcal{Q}_{i}}{r}, \quad \mathcal{Q}_{i}=\mu \sinh ^{2} \delta_{i}
$$

for the constituent D2-branes, and

$$
H_{i}=1+\frac{\mathcal{P}_{i}}{r}, \quad \mathcal{P}_{i}=\mu \sinh ^{2} \gamma_{i}
$$


for the constituent D4-branes.

(2) In the expression for the field strength $\mathcal{F}_{4}$ of the three-form field, we make the following replacements:

$$
H_{i}^{\prime} \rightarrow H_{i}^{\prime}=1+\frac{Q_{i}}{r+\mathcal{Q}_{i}-Q_{i}}=\left[1-\frac{Q_{i}}{r} H_{i}^{-1}\right]^{-1}, \quad Q_{i}=\mu \sinh \delta_{i} \cosh \delta_{i},
$$

for the electric (D2-brane) part, and

$$
H_{i} \rightarrow H_{i}^{\prime}=1+\frac{P_{i}}{r}, \quad P_{i}=\mu \sinh \gamma_{i} \cosh \gamma_{i},
$$

for the magnetic (D4-brane) part. Here $Q_{i}$ and $P_{i}$ are the electric and magnetic charges respectively. The BPS limit is realized when $\mu \rightarrow 0, \delta_{i} \rightarrow \infty$, and $\gamma_{i} \rightarrow \infty$, while the charges $Q_{i}$ and $P_{i}$ are kept fixed. In this case $\mathcal{Q}_{i}=Q_{i}$ and $\mathcal{P}_{i}=P_{i}$, so that $H_{i}^{\prime}=H_{i}$. We note that the metric of two intersecting D-branes are not extremal in the BPS limit.

(3) In the case when the solution has a null isometry, i.e. the intersecting D-branes have a common string along some direction $y$, one can add momentum along $y$ by applying the coordinate transformation

$$
t^{\prime}=\cosh \sigma t-\sinh \sigma y, \quad y^{\prime}=-\sinh \sigma t+\cosh \sigma y
$$

to the non-extremal background which is obtained according to the above two steps. Then we obtain that

$$
\begin{aligned}
-f(r) d t^{2}+d y^{2} & \rightarrow-f(r) d t^{\prime 2}+d y^{\prime 2} \\
& =-d t^{2}+d y^{2}+\frac{\mu}{r}(\cosh \sigma d t-\sinh \sigma d y)^{2},
\end{aligned}
$$

where $\sigma$ is a boost parameter. This transformation is called the boost transformation.

\section{SURFACE GRAVITY AND COMPACTIFICATION}

In ten-dimensions [2,6,7], the metrics have the compactification radii. The radii are usually taken to be arbitrary. However for the generic radii, we find that the Euler numbers of the metrics are non-integers. In this section, we consider the surface gravities of the compactified dimensions. We constrain the compactification radii using the surface gravity. As a result, we obtain the integer Euler numbers.

We consider a way to constrain the radii in the compactified directions. We first consider the radius in the Euclidean time direction. We treat the Euclidean time coordinate as the polar angle in the $t-r$ directions. We define the deficit angle $\delta$ in the $t-r$ directions as,

$$
\frac{1}{2} \int_{0}^{\beta} d t \int_{r_{H}}^{\infty} d r \sqrt{g_{t t} g_{r r}} R_{(2)}=2 \pi-\delta
$$

where $R_{(2)}$ is the scalar curvature in the $t-r$ directions. $\beta$ is the period of the time

coordinate. $r_{H}$ is the radius of the event horizon, which satisfies that $\left.g_{t t}\right|_{r=r_{H}}=0$. The metric in the $t-r$ directions is 


$$
d s_{(2)}^{2}=g_{t t} d t^{2}+g_{r r} d r^{2}
$$

The scalar curvature $R_{(2)}$ and the extrinsic curvature $K$ are

$$
R_{(2)}=-\frac{1}{\sqrt{g_{t t} g_{r r}}} \partial_{r}\left[\frac{1}{2} \frac{\partial_{r} g_{t t}}{\sqrt{g_{t t} g_{r r}}}\right], \quad K=\frac{1}{\sqrt{g_{r r}}}\left[\frac{1}{2} \frac{\partial_{r} g_{t t}}{g_{t t}}\right] .
$$

We define the Euler number $\chi$ from the Gauss-Bonnet theorem is

$$
\begin{aligned}
2 \pi \chi & =\frac{1}{2} \int_{0}^{\beta} d t \int_{r_{H}}^{\infty} d r \sqrt{g_{t t} g_{r r}} R_{(2)}-\left.\int_{0}^{\beta} d t \sqrt{g_{t t}} K\right|_{r=\infty} \\
& =\left.\frac{\beta}{2} \frac{\partial_{r} g_{t t}}{\sqrt{g_{t t} g_{r r}}}\right|_{r=r_{H}} .
\end{aligned}
$$

If no singular effect of the event horizon exists, then the topology in $t-r$ directions is $\mathbf{R}^{\mathbf{2}}$, then the Euler number is 1 . Therefore, from (20) the radius $\beta$ in the time direction is

$$
\beta=\frac{2 \pi}{\kappa_{t}},\left.\quad \kappa_{t} \equiv \frac{1}{2} \frac{\partial_{r} g_{t t}}{\sqrt{g_{r r} g_{t t}}}\right|_{r=r_{H}},
$$

where $\kappa$ is the surface gravity. $r_{H}$ is a radius of the black hole event horizon. We obtain that the deficit angle is 0 , and the Euler number is 1 . Then if we use the radius $\beta$ in the time direction, the singular effect of the event horizon $r_{H}$ is absent. Therefore we take this radius in the time direction to obtain the smooth geometry around the event horizon.

For example, we find that the Euler numbers of the Schwarzschild metric, the KerrNewman metric, and the $U(1)$ dilaton metric to be integers by using the relation between the surface gravity and the compactification radius (the inverse temperature).

We extend this idea to the ten-dimensional manifolds in order to obtain the integer Euler numbers. We next consider the compactification radii of the black holes in ten-dimensions. We treat the black holes which have six compactified directions. These compactified directions are the same as the Euclidean time direction, then the topology is $\mathbf{R}^{\mathbf{8}} \times \mathbf{S}^{\mathbf{2}}$. The metric $g_{i i}$ of the compactified directions does not depend the variables of the compactified directions. Therefore we can choose one of the compactified directions and consider the Euler numbers in the $i-r$ directions partly.

We consider the compactification radii in the $i-r$ directions, where $i$ is one of the compactified directions. The topologies of the $i-r$ directions are $\mathbf{R}^{\mathbf{2}}$. We define the deficit angle $\delta_{i}$ in the $i-r$ directions as

$$
\frac{1}{2} \int_{0}^{\beta_{i}} d x^{i} \int_{r_{H}}^{\infty} d r \sqrt{g_{i i} g_{r r}} R_{(2)}=2 \pi-\delta_{i}
$$

where $R_{(2)}$ is the scalar curvature in the $i-r$ directions. $\beta_{i}$ are the periods of the $i$-th coordinate. The metric in the $i-r$ directions is

$$
d s_{(2)}^{2}=g_{i i} d t^{2}+g_{r r} d r^{2}
$$

The scalar curvature $R_{(2)}$ and the extrinsic curvature $K$ are

$$
R_{(2)}=-\frac{1}{\sqrt{g_{i i} g_{r r}}} \partial_{r}\left[\frac{1}{2} \frac{\partial_{r} g_{i i}}{\sqrt{g_{i i} g_{r r}}}\right], \quad K=\frac{1}{\sqrt{g_{r r}}}\left[\frac{1}{2} \frac{\partial_{r} g_{i i}}{g_{i i}}\right] .
$$


We define the Euler number $\chi$ from the Gauss-Bonnet theorem as

$$
\begin{aligned}
2 \pi \chi & =\frac{1}{2} \int_{0}^{\beta_{i}} d x^{i} \int_{r_{H}}^{\infty} d r \sqrt{g_{i i} g_{r r}} R_{(2)}-\left.\int_{0}^{\beta_{i}} d x^{i} \sqrt{g_{i i}} K\right|_{r=\infty} \\
& =\left.\frac{\beta}{2} \frac{\partial_{r} g_{i i}}{\sqrt{g_{i i} g_{r r}}}\right|_{r=r_{H}} .
\end{aligned}
$$

If no singular effect of the event horizon exists, then the topology in $i-r$ directions is $\mathbf{R}^{\mathbf{2}}$, and the Euler number is 1 . Therefore, from (21) we suppose that the compactification radius $\beta_{i}$ in the $i$-th direction is defined by the following "surface gravity $\kappa_{i}$ ":

$$
\begin{aligned}
\beta_{i}\left(r_{H}\right)= & \frac{2 \pi}{\kappa_{i}},\left.\quad \kappa_{i}\left(r_{H}\right) \equiv \frac{1}{2} \frac{\partial_{r} g_{i i}}{\sqrt{g_{r r} g_{i i}}}\right|_{r=r_{H}}, \\
& (i=1 \cdots 6) .
\end{aligned}
$$

We obtain that the vanishing deficit angles in the compactified directions. Therefore we use this radius $\beta_{i}$ in the compactified directions to obtain the smooth geometry around the event horizon.

We obtain the integer Euler numbers for the ten-dimensional manifolds using these compactification radii. For example, let us consider the following metric:

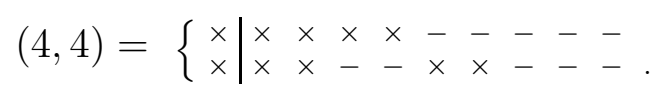

In the non-extremal version of this metric, the event horizon is located at

$$
r_{H}=\mu \text {. }
$$

In the third direction, the compactification radius is

$$
\beta_{3}\left(r_{H}\right)=4 \pi\left(\mu+\mathcal{M}_{1}\right)\left(\mu+\mathcal{M}_{2}\right)^{3 / 2} /\left(\mathcal{M}_{1}-\mathcal{M}_{2}\right) \sqrt{\mu}
$$

Using this definition, we indeed find that the Euler number of this example to be an integer as follows:

$$
\begin{aligned}
\chi= & \frac{1}{(4 \pi)^{10} 10 !} \int d x^{10} \epsilon^{a b c d e f g h i j} R_{a b} R_{c d} R_{e f} R_{g h} R_{i j} \\
= & \frac{1}{(4 \pi)^{10}} \int_{r_{H}}^{\infty} d r \int_{0}^{\beta_{t}(r)} d t \int_{0}^{2 \pi} d \phi \int_{0}^{\pi} d \theta \sin \theta \\
& \times \prod_{i=1}^{6} \int_{0}^{\beta_{i}(r)} d x_{i} R_{t r} R_{\theta \phi} R_{12} R_{34} R_{56} \\
= & 2 .
\end{aligned}
$$

We further obtain the same Euler number for all the other metrics which are related to this metric by T-duality as it will be explained in the next section. We emphasize again that the Euler numbers are non-integers if we consider the compactification radii as arbitrary constants. 


\section{BLACK HOLE ENTROPIES AND T-DUALITY}

In this section, we study the entropies of the intersecting D-branes using the compactification radii as defined in the previous section. The entropies are calculated semiclassically by using $S=A / 4 G$ in this section. Here $A$ is the area of the event horizon, and $G$ is the Newton's constant. We obtain the entropies which are T-duality invariant. In the BPS limits $(\mu \rightarrow 0)$, we obtain the finite and non-vanishing entropies only for two intersecting D-branes. They are found to be proportional to the product of the charges of each D-brane. We further study the entropies of boosted metrics. We obtain the relations between the entropies and the internal momenta of the intersecting D-branes. These relations are the same as that of the microscopic D-brane picture [3] 5 .

We first study the entropies of the metric with the boost parameter $\sigma=0$ in (19). We define the proper length in the $i$-th direction as:

$$
\begin{aligned}
L_{i}(r) \equiv & \left|\int_{0}^{\beta_{i}(r)} \sqrt{g_{i i}} d x_{i}\right|=4 \pi\left|\frac{\sqrt{g_{r r}}}{\partial_{r}\left(\ln g_{i i}\right)}\right|, \\
& (i=1, \cdots, 6) .
\end{aligned}
$$

Using these definitions, the entropies of the black holes are

$$
S=A_{8} /\left.4 G_{10}\right|_{r=\mu}=L_{1} L_{2} L_{3} L_{4} L_{5} L_{6} A_{\theta \phi} /\left.4 G_{10}\right|_{r=\mu}
$$

Here $A_{8}$ is the area of the event horizon, $A_{\theta \phi}$ is the area in the $\theta-\phi$ directions.

For example, we consider the following metric :

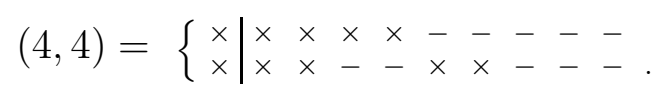

In the third direction, the proper length is

$$
L_{3}=\int_{0}^{\beta_{3}} \sqrt{g_{33}} d x_{3}=4 \pi\left(r+\mathcal{M}_{1}\right)^{5 / 4}\left(r+\mathcal{M}_{2}\right)^{5 / 4} /\left(\mathcal{M}_{1}-\mathcal{M}_{2}\right) \sqrt{r},
$$

where $\mathcal{M}_{i}$ are $\mathcal{Q}_{i}$ or $\mathcal{P}_{i}$. With this definition, we find the following relations :

$$
\begin{aligned}
L_{3}=L_{4}=L_{5}=L_{6} & =4 \pi\left(r+\mathcal{M}_{1}\right)^{5 / 4}\left(r+\mathcal{M}_{2}\right)^{5 / 4} /\left(\mathcal{M}_{1}-\mathcal{M}_{2}\right) \sqrt{r} \\
& \equiv L_{a} \\
L_{1}=L_{2} & =4 \pi\left(r+\mathcal{M}_{1}\right)^{1 / 4}\left(r+\mathcal{M}_{2}\right)^{1 / 4} \sqrt{r} \\
& \equiv L_{b} .
\end{aligned}
$$

Let us consider the proper lengths of the metrics which are dual to each other. If we apply the T-duality transformation to the fourth-direction of $(4,4)$ type metric, we obtain the $(3,5)$ type metric,

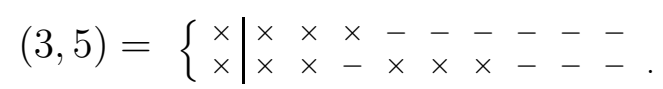

For this metric, we also obtain the proper lengths which are the same with (23). Then the entropies of $(3,5)$ type metrics are the same with $(4,4)$ type metrics. We also obtain the 
identical proper lengths for all the other metrics which are dual to each other. Therefore the entropies of the T-dual metrics are the same.

We can also obtain the analogous relations with the different numbers of the D-branes. The area in the $\theta-\phi$ directions is

$$
A_{\theta \phi}=\int_{0}^{\pi} d \theta \int_{0}^{2 \pi} d \phi \sin \theta \sqrt{g_{\theta \theta} g_{\phi \phi}}=4 \pi\left(r+\mathcal{M}_{1}\right)^{1 / 2}\left(r+\mathcal{M}_{2}\right)^{1 / 2} r .
$$

Then we find that the entropy of two intersecting D-branes is given by

$$
\begin{aligned}
S= & A_{8} /\left.4 G_{10}\right|_{r=\mu}=L_{a}^{4} L_{b}^{2} A_{\theta \phi} /\left.4\right|_{r=\mu} \\
= & (4 \pi)^{7}\left(\mu+\mathcal{M}_{1}\right)^{8}\left(\mu+\mathcal{M}_{2}\right)^{8} \\
& /\left[\left(\mathcal{M}_{1}-\mathcal{M}_{2}\right)^{4}\left[\mu\left(\mathcal{M}_{1}+\mathcal{M}_{2}\right)+2 \mathcal{M}_{1} \mathcal{M}_{2}\right]^{2}\right],
\end{aligned}
$$

where $G_{10}$ is the ten-dimensional Newton's constant. For three intersecting D-branes, the entropy is given by

$$
\begin{aligned}
S= & A_{8} /\left.4 G_{10}\right|_{r=\mu} \\
= & (4 \pi)^{7}\left(\left(\mu+\mathcal{M}_{1}\right)^{8}\left(\mu+\mathcal{M}_{2}\right)^{8}\left(\mu+\mathcal{M}_{3}\right)^{8} \mu^{2}\right. \\
& /\left[\left[\mu^{2}\left(\mathcal{M}_{1}+\mathcal{M}_{2}-\mathcal{M}_{3}\right)+2 \mu \mathcal{M}_{1} \mathcal{M}_{2}+\mathcal{M}_{1} \mathcal{M}_{2} \mathcal{M}_{3}\right]\right. \\
& \times\left[\mu^{2}\left(\mathcal{M}_{2}+\mathcal{M}_{3}-\mathcal{M}_{1}\right)+2 \mu \mathcal{M}_{2} \mathcal{M}_{3}+\mathcal{M}_{1} \mathcal{M}_{2} \mathcal{M}_{3}\right] \\
& \left.\times\left[\mu^{2}\left(\mathcal{M}_{3}+\mathcal{M}_{1}-\mathcal{M}_{2}\right)+2 \mu \mathcal{M}_{3} \mathcal{M}_{1}+\mathcal{M}_{1} \mathcal{M}_{2} \mathcal{M}_{3}\right]\right]^{2} .
\end{aligned}
$$

For four intersecting D-branes, the entropy is

$$
\begin{aligned}
S= & A_{8} /\left.4 G_{10}\right|_{r=\mu} \\
= & (4 \pi)^{7}\left(\left(\mu+\mathcal{M}_{1}\right)^{8}\left(\mu+\mathcal{M}_{2}\right)^{8}\left(\mu+\mathcal{M}_{3}\right)^{8}\left(\mu+\mathcal{M}_{4}\right)^{8}\right. \\
& / \mu^{6}\left[\left[\mu^{2}\left(\mathcal{M}_{1}+\mathcal{M}_{2}-\mathcal{M}_{3}-\mathcal{M}_{4}\right)+2 \mu\left(\mathcal{M}_{1} \mathcal{M}_{2}-\mathcal{M}_{3} \mathcal{M}_{4}\right)\right.\right. \\
& \left.+\left(\mathcal{M}_{1}+\mathcal{M}_{2}\right) \mathcal{M}_{3} \mathcal{M}_{4}-\left(\mathcal{M}_{3}+\mathcal{M}_{4}\right) \mathcal{M}_{1} \mathcal{M}_{2}\right] \\
& \times\left[\mu^{2}\left(\mathcal{M}_{1}+\mathcal{M}_{3}-\mathcal{M}_{2}-\mathcal{M}_{4}\right)+2 \mu\left(\mathcal{M}_{1} \mathcal{M}_{3}-\mathcal{M}_{2} \mathcal{M}_{4}\right)\right. \\
& \left.+\left(\mathcal{M}_{1}+\mathcal{M}_{3}\right) \mathcal{M}_{2} \mathcal{M}_{4}-\left(\mathcal{M}_{2}+\mathcal{M}_{4}\right) \mathcal{M}_{1} \mathcal{M}_{3}\right] \\
& \times\left[\mu^{2}\left(\mathcal{M}_{1}+\mathcal{M}_{4}-\mathcal{M}_{2}-\mathcal{M}_{3}\right)+2 \mu\left(\mathcal{M}_{1} \mathcal{M}_{4}-\mathcal{M}_{2} \mathcal{M}_{3}\right)\right. \\
& \left.\left.+\left(\mathcal{M}_{1}+\mathcal{M}_{4}\right) \mathcal{M}_{2} \mathcal{M}_{3}-\left(\mathcal{M}_{2}+\mathcal{M}_{3}\right) \mathcal{M}_{1} \mathcal{M}_{4}\right]\right]^{2} \cdot
\end{aligned}
$$

We also list the entropy formula for single brane:

$$
S=A_{8} /\left.4 G_{10}\right|_{r=\mu}=(4 \pi)^{7} \mu^{6}\left(\mu+\mathcal{M}_{1}\right)^{8} / \mathcal{M}_{1}^{6} .
$$


If we consider the BPS limit (namely $\mu \rightarrow 0$ ), we can obtain the finite and non-vanishing entropies only for the metrics of two intersecting D-branes. In order to interpret these entropies, we define the quantized D-brane charges in the wrapping directions except for the intersecting directions. They are the integer numbers. In the case of $(4,4)$ case, we define the quantized magnetic charges of the D4-branes as

$$
\begin{aligned}
N_{1} & =\left.\frac{L_{a}^{2}}{4 \pi} \int \mathcal{F}_{\theta \phi 12} d \Omega\right|_{r=1} \\
& =(4 \pi)^{2} \mathcal{M}_{1}\left(\mathcal{M}_{1} \mathcal{M}_{2}\right)^{5 / 2} /\left(\mathcal{M}_{1}-\mathcal{M}_{2}\right)^{2} \\
N_{2} & =\left.\frac{L_{a}^{2}}{4 \pi} \int \mathcal{F}_{\theta \phi 12} d \Omega\right|_{r=1} \\
& =(4 \pi)^{2} \mathcal{M}_{2}\left(\mathcal{M}_{1} \mathcal{M}_{2}\right)^{5 / 2} /\left(\mathcal{M}_{1}-\mathcal{M}_{2}\right)^{2}
\end{aligned}
$$

Using these charges, we find the entropies as

$$
S=(4 \pi)^{3} N_{1} N_{2} / 4
$$

In the T-dual cases of this metric, $(3,5)$ and $(2,6)$, we define

$$
N= \begin{cases}\frac{L_{a}^{2}}{4 \pi} \int * \mathcal{F} & \text { (electric), } \\ \frac{L_{a}^{2}}{4 \pi} \int \mathcal{F} & \text { (magnetic) }\end{cases}
$$

where $*$ is Hodge dual in four-dimensions. Then we find that the entropies for all metrics of two intersecting D-branes as the same with (24). Therefore we observe that the entropies of two intersecting D-branes are the product of the quantized charges of each D-brane.

Next we consider the case of the large boost parameter $(\sigma \gg 1)$. In this case, the metric becomes

$$
\begin{aligned}
-f(r) d t^{2}+d y^{2} & \rightarrow-f(r) d t^{\prime 2}+d y^{\prime 2} \\
& =-d t^{2}+d y^{2}+\frac{\mu}{r}(\cosh \sigma d t-\sinh \sigma d y)^{2} \\
& \sim-d t^{2}+d y^{2}+\frac{\mu \cosh ^{2} \sigma}{r}(d t-d y)^{2} .
\end{aligned}
$$

Here we have introduced $\mu^{\prime}=\mu \cosh ^{2} \sigma$. Then the event horizon is at $r_{H}=\mu^{\prime}$. The proper length in the $y$-direction is

$$
L_{y}=4 \pi\left[\left(\mathcal{M}_{1}+r\right)\left(\mathcal{M}_{2}+r\right)\right]^{5 / 4} \sqrt{r} /\left(2 r^{2}-2 \mathcal{M}_{1} \mathcal{M}_{2}\right) .
$$

For $(3,3),(2,4)$ and $(1,5)$ cases, we find the following entropy formula as

$$
S=L_{a}^{4} L_{b} L_{y} A_{\theta \phi} /\left.4\right|_{r=\mu^{\prime}}
$$

In the $(4,4),(3,5)$ and $(2,6)$ cases, we have two isometric directions. If we consider the case that only one of these directions is boosted, the entropy is given by

$$
S=L_{a}^{4} L_{b} L_{y} A_{\theta \phi} /\left.4\right|_{r=\mu^{\prime}}
$$


We may further consider the case that the metric is boosted in two different directions. Let us assume that the $y_{2}$ direction is boosted first and then the $y_{1}$ direction is boosted. After such a process, we obtain the following metric as

$$
\begin{aligned}
-f(r) d t^{2}+d y_{1}^{2}+d y_{2}^{2} \rightarrow & -f(r) d t^{\prime 2}+d y_{1}^{\prime 2}+d y_{2}^{\prime 2} \\
= & -d t^{2}+d y_{1}^{2}+d y_{2}^{2} \\
& +\frac{\mu}{r}\left(\cosh ^{2} \sigma_{1} \cosh ^{2} \sigma_{2} d t-\sinh \sigma_{1} d y_{1}-\cosh ^{2} \sigma_{1} \sinh ^{2} \sigma_{2} d y_{2}\right)^{2} \\
\sim & -d t^{2}+d y_{1}^{2}+d y_{2}^{2}+\frac{\mu \cosh ^{2} \sigma_{1} \cosh ^{2} \sigma_{2}}{r}\left(d t-d y_{2}\right)^{2} .
\end{aligned}
$$

Therefore we obtain the same metric with the boost in the single direction. As a result, we find that the entropies of the boosted metrics are the same.

According to the definitions in [3 5], we define the quantized internal momentum $p$ and the charge $n$ in the direction of $y$. Here $n$ is a number of the state level of the effective conformal field theory in the D-brane picture. The momentum $p$ is quantized in term of the surface area of the event horizon $A_{8}$. We define the charge and the momentum as

$$
\begin{aligned}
n & =L_{y} p \\
n & =\int d A_{8} \mu^{\prime} /\left.r\right|_{r=\mu^{\prime}} \\
& =L_{a}^{4} L_{b} L_{y} A_{\theta \phi} \\
& \sim\left(\mathcal{M}_{1} \mathcal{M}_{2}\right)^{6} /\left(\mathcal{M}_{1}-\mathcal{M}_{2}\right)^{4} .
\end{aligned}
$$

Using these quantities, we obtain the entropies in the BPS limit with $\sigma \gg 1$ as follows,

$$
S \sim \sqrt{N_{1} N_{2} n}
$$

This entropy formula of the boosted metrics is the same with that of the microscopic D-brane picture [3].

\section{CONCLUSION}

We have studied the Euler numbers and the entropies of the non-extremal black holes which are constructed by the intersecting D-branes in ten-dimensions. The Euler numbers are generally integers. In ten-dimensions, the metrics have the arbitrary compactification radii. However for the generic radii, the Euler numbers of the metrics are non-integers. To avoid these difficulties, we need the way to constrain the compactification radii.

The another physical reasoning that we need to fix the compactification radii is in order to avoid the singular effects of the horizon in the compactified directions. We have discussed this point in the section 3 . We consider the way to define the period in the time directions. If no singular effects of the horizon exist in $t-r$ directions, then the topology of these directions are $\mathbf{R}^{\mathbf{2}}$, and the Euler number of these directions is 2. Using the Gauss-Bonnet theorem, we find that the Euclidean time coordinate have the period which is the inverse of the surface gravity. Therefore we need to take this period in the time direction to avoid the singular effects of the horizon. Similarly, we need to fix the compactification radii to 
avoid the singular effects of the horizon in the compactified directions. We find the necessity that the compactification radii are the inverses of the surface gravity in the compactified directions using the Gauss-Bonnet theorem.

We identify the inverse of the surface gravities as the compactification radii in order to obtain the integer Euler numbers. The entropies of the black holes are T-duality invariant when we use the compactification radii which are defined by surface gravities. In the BPS limit, we have the finite and non-zero entropies only with two intersecting D-branes. We have introduced the quantized D-brane charges $N_{1}$ and $N_{2}$. We find the common relation of these entropies as $S \sim N_{1} N_{2}$, when the boost parameter vanishes. Therefore we observe that the entropies are proportional to the product of the charges of each D-brane.

In the case of the large boost parameter, we also obtain the entropy formula for the black holes constructed by two intersecting D-branes. The entropies of the boosted black holes are also T-duality invariant. We have introduced the quantum number $n$. Here $n$ is a number of the state level of the effective conformal field theory in the D-brane picture. The entropies are $S \sim \sqrt{N_{1} N_{2} n}$. The entropies of the boosted black holes can be interpreted as the entropies of the microscopic states in the D-brane picture.

We need to consider the microscopic interpretation of the entropies in association with the non-boosted black holes in the D-brane picture. The entropies of the non-boosted black holes reduce to $S \sim N_{1} N_{2}$. The black holes have no momenta $n$ in this limit. On the other hand, the entropies in the microscopic D-brane picture $S \sim \sqrt{N_{1} N_{2} n}$ are obtained for the large momenta. Therefore the entropy formula for the non-boosted black holes could be entirely different from that for the boosted black holes which agrees with the microscopic Dbrane picture. The microscopic entropies in the D-brane picture with small or zero momenta were not discussed so far. We further need to study the microscopic interpretation of the entropies with the momenta $n \sim 0$ in the D-brane picture.

We have proposed a method to constrain the compactification radii of the non-extremal black holes of the intersecting D-branes. We have identified the inverse of the surface gravities as the compactification radii. We have correctly obtained the integer Euler numbers of the black holes. Although we do not know another way to constrain the compactification radii, we have not shown that the way to constrain the compactification radii is unique. Therefore another way to constrain the compactification radii might be found. We further need to study the way and the physical reasoning to constrain the compactification radii.

\section{ACKNOWLEDGMENTS}

We thank Y. Kitazawa for discussions and for carefully reading the manuscript and suggesting various improvements. 


\section{REFERENCES}

[1] A. Sen, Mod.Phys.Lett. A10, 208 (1995);

M. Cvetic and D. Youm, Phys.Rev. D53, 584 (1995)

[2] M. Cvetic and A.A. Tseytlin, Nucl.Phys. B478, 181 (1996)

[3] A. Strominger and C. Vafa, Phys.Lett. B379, 99 (1996)

[4] G.T. Horowitz and A. Strominger, Phys.Rev.Lett. 77, 2368 (1996)

[5] J.M. Maldacena and A. Strominger, Phys.Rev. D55, 861 (1997)

[6] A.A. Tseytlin, Nucl.Phys. B475,149 (1996);

I.R. Klebanov and A.A. Tseytlin, Nucl.phys. B475, 179 (1996)

[7] J.P. Gauntlett and D.A. Kastor and J. Traschen, Nucl.Phys. B478, 544 (1996)

[8] E. Bergshoeff, M.de Roo, E. Eyras, B. Janssen, J.P.van der Schaar, Nucl.Phys. B494, 119 (1997)

[9] S.W. Hawking and G.T. Horowitz and S.F. Ross, Phys.Rev. D51, 4302 (1995)

[10] G.W. Gibbons and R.E. Kallosh, Phys.Rev. D51, 2839 (1995)

[11] S. Liberati and G. Polliofrone, Nucl.Phys.Proc.Suppl. 57, 197 (1997);

S. Liberati, Nuovo.Cim. 112B, 405 (1997);

K. Suzuki, preprint hep-th/9611095 